\title{
The Role of Alpha-Lipoic Acid in the Pathomechanism of Acute Ischemic Stroke
}

\author{
Qingqing Wang Chengmei Lv Yongxin Sun Xu Han Shan Wang \\ Zhengchun Mao Yanming Xin Bing Zhang \\ Department of Anesthesiology, Second Affiliated Hospital of Harbin Medical University, Harbin, China
}

\author{
Key Words \\ Stroke $\bullet$ Inflammation $\bullet \alpha$ - lipoic acid $\bullet$ Microglia polarization $\bullet$ NF-kB
}

\begin{abstract}
Background/Aims: Ischemic stroke results in increased cerebral infarction, neurological deficits and neuroinflammation. The underlying mechanisms involving the anti-inflammatory and neuroprotective properties of $\alpha$-Lipoic acid ( $\alpha$-LA) remain poorly understood. Herein, we investigated the potential role of $\alpha-\mathrm{LA}$ in a middle cerebral artery occlusion (MCAO) rat model and an in vitro lipopolysaccharide (LPS)-induced microglia inflammation model. Methods: In the in vivo study, infarct volume was examined by TTC staining and Garcia score was used to evaluate neurologic recovery. The cytokines were evaluated by enzyme-linked immunosorbent assay, and protein expression of microglia phenotype and NF-KB were measured using western blot. In the in vitro study, the expressions of microglia M1/M2 phenotype were evaluated using qRT-PCR, and immunofluorescence staining was used to assess the nuclear translocation of NF-KB. Results: Both $20 \mathrm{mg} / \mathrm{kg}$ and $40 \mathrm{mg} / \mathrm{kg}$ of $\alpha-\mathrm{LA}$ alleviated infarct size, brain edema, and neurological deficits. Furthermore, $\alpha$-LA induced the polarization of microglia to the M2 phenotype, modulated the expression of IL-1 $\beta$, IL-6, TNF- $\alpha$ and IL-10, and attenuated the activation of NF-KB after MCAO. $\alpha$-LA inhibited the expression of M1 markers, increased activation of the M2 markers, and suppressed the nuclear translocation of NF-KB in LPSstimulated BV2 microglia. Conclusions: $\alpha$-LA improved neurological outcome in experimental stroke via modulating microglia M1/M2 polarization. The potential mechanism of $\alpha$-LA might be mediated by inhibition of NF-KB activation via regulating phosphorylation and nuclear translocation of $\mathrm{p} 65$.
\end{abstract}

(C) 2018 The Author(s)

Published by S. Karger AG, Basel

\section{Introduction}

Stroke is a leading cause of long-term disability and death worldwide, ranking second to cardiac ischemia [1]. Thrombolytic therapy with tissue-type plasminogen activator is the only treatment approved by United States FDA in acute ischemic stroke [2]. However, due to the narrow thrombolytic therapeutic time window $(4.5 \mathrm{~h})$, few patients benefit from 
thrombolytic treatment. Recently, several cell-based and pharmacological therapies, such as bone marrow derived mesenchymal stromal cells, atorvastatin, candesartan, minocycline were studied for stroke $[3,4]$. But until recently, there is still no effective treatment that can increase the survival rate or improve the quality of life after ischemic stroke. Therefore, it is necessary to establish other therapies for ischemic stroke.

$\alpha$-Lipoic acid ( $\alpha$-LA) has been used in the treatment of diabetes for several years [5]. Choi et al [6]. demonstrated that use of $\alpha$-LA in patients with acute ischemic stroke and diabetes who are treated with tPA is associated with favorable outcomes both at 3 months and at 1 year. However, the underlying mechanisms of $\alpha$-LA are not fully established. As a strong antioxidant, it has been reported that $\alpha$-LA is able to repair oxidative damage, scavenge the reactive oxygen species (ROS), and regenerate endogenous antioxidants [7, 8]. Besides, recent evidences suggest that $\alpha$-LA may confer neuroprotection through antiinflammation effects in multiple sclerosis [9], stroke [10] and macrophages in vitro [11]. However, the underlying mechanisms involving the anti-inflammatory and neuroprotective properties of $\alpha$-LA in stroke remain poorly understood.

The inflammatory response plays an important role in the pathogenesis of stroke [12]. Upregulation of pro-inflammatory mediators e.g. interleukin (IL)-1 $\beta$, IL- 6 and tumor necrosis factor (TNF)- $\alpha$ during the course of ischemia reperfusion induces neutrophil infiltration into the penumbra in a time-dependent manner, leading to serious neurological dysfunction [13]. Nuclear factor (NF)- $\kappa B$ and microglia activation has been shown to play an important role in cerebral injury induced by experimental stroke [14]. Microglia is the predominant inflammatory cell responsible for inflammation in the brain, and can be polarized into the "classically activated" M1 phenotype or the "alternatively activated" M2 phenotype [15]. Many studies have shown that activated microglia in M1 state exhibits harmful properties which characterized by the production of pro-inflammatory cytokines such as IL- $1 \beta$, IL-6, TNF- $\alpha$. In contrast, microglia in M2 state demonstrates protective and reparative functions including the fine-tuning of inflammation $[16,17]$. It also has been proved that the inflammation reaction can be inhibited and the neurological outcome can be improved by modulating the phenotype of microglia [16].

The role of $\alpha$-LA in the regulation of microglial phenotypes remains to be elucidated. Therefore, the present study aimed to investigate the effect of $\alpha$-LA on neurological outcomes in a middle cerebral artery occlusion (MCAO) rat model, and to evaluate whether the neuroprotective effects were associated with suppression of neuroinflammation and regulating microglia M1/M2 polarization.

\section{Materials and Methods}

\section{Animals}

All experiments were approved by the Institutional Animal Care and Use Committee of Harbin Medical University in Harbin, Heilongjiang, China. This study was performed using male Sprague-Dawley rats weighing 220-250 g. Food and water was available ad libitum until the morning of the experiment. All studies involving animals are reported in accordance with the ARRIVE guidelines for reporting experiments involving animals [18, 19].

\section{Transient middle cerebral artery occlusion (MCAO)}

Anesthesia was performed with $5 \%$ sevoflurane, and rats were maintained with $3 \%$ sevoflurane in $30 \%$ oxygen. Spontaneous breath was allowed throughout the experiment under an oxygen flow rate of 2 $\mathrm{L} / \mathrm{min}$. Body temperature was monitored continuously with a rectal probe and maintained at $37 \pm 0.5{ }^{\circ} \mathrm{C}$ using a heating lamp. A silicon-coated nylon monofilament (tip diameter: $0.37 \pm 0.02 \mathrm{~mm}$, Doccol, MA, USA) was inserted into the middle cerebral artery as described previously [20]. Cerebral blood flow was assessed by Laser Doppler Flowmetry (TF5000; PERIMED AB; Stockholm; Sweden) [21]. Two hours after occlusion, the filament was withdrawn for reperfusion. Sham-operated rats underwent all aspects of MCAO surgeries 


\section{Cellular Physiology Cell Physiol Biochem 2018;48:42-53 and Biochemistry Published online: July 11, $2018 \quad \begin{aligned} & \text { DOI: 10.1159/000491661 } 2018 \text { The Author(s). Published by S. Karger AG, Basel } \\ & \text { www.karger.com/cpb }\end{aligned}$}

Wang et al.: A-LA Induces Microglia to M2 Phenotype in Stroke

including midline incision and exposing of arteries but without insertion of the filament. Animals were excluded from the study based on cerebral blood flow (CBF) data. In the MCAO group, rats with a CBF drop less than $70 \%$ of the baseline were excluded.

\section{Animal groups}

At the same time of reperfusion, rats were randomly divided into 3 groups: LA20, LA40, and control group, and received injections of 20, $40 \mathrm{mg} / \mathrm{kg} \alpha$-LA (Sigma-Aldrich, MO, USA) or an equal volume of saline via the external jugular vein, respectively. The dose used in this study was based on the data obtained in our preliminary study showing that treatment with $20-40 \mathrm{mg} / \mathrm{kg} \alpha$-LA exerted obvious neuroprotective effects. All experiments were carried out randomly by researchers blinded to the grouping. Among the total 45 rats subjected to MCAO performance, 5 were excluded before testing because of an unqualified drop of cerebral blood flow.

\section{Assessment of infarction volume}

Rats were euthanized at $24 \mathrm{~h}$ after reperfusion. Their brains were removed and the forebrains were cut into 6 coronal sections (2-mm-thick). The brain sections were then incubated in $1 \%$ solution of 2, 3, 5-triphenyltetrazolium chloride (TTC) (Sigma-Aldrich, MO, USA) at $37^{\circ} \mathrm{C}$ for $10 \mathrm{~min}$ [22]. The area of each section was measured using Image J software (NIH, Bethesda, MD, USA).

\section{Behavioral Testing}

All animals were evaluated before the experiment to ensure normal neurological function. The neurological deficits were evaluated using the 18-point Garcia score system [23] at $24 \mathrm{~h}$ after MCAO. The examination can comprehensively evaluate neurological deficits including both motor and sensory function in rat MCAO model, and presents a high relativity with focal brain injury. Briefly, the sensorimotor function of each rat was assessed based on spontaneous activity, symmetry of limbs, forepaw outstretching, climbing, body proprioception, and response to vibrissae touch.

\section{Western blotting for assessment of microglia polarization and NF- $\kappa B$ activation}

Brain tissue samples of the ipsilateral penumbra [24] were obtained at $24 \mathrm{~h}$ after reperfusion and homogenized in loading buffer (Beyotime, Jiangsu, China). The homogenates were then centrifuged at 13, $200 \mathrm{~g}$ for 15 min at $4{ }^{\circ} \mathrm{C}$ (5407B00304, Eppendorf, Hamburg, Germany), and supernatants were collected. Nuclear and cytosolic proteins were extracted using a modified method described previously [25]. Protein concentration in each sample was determined using a BCA protein assay kit (Beyotime, Jiangsu, China). Equivalent amounts of protein for each sample was separated using SDS-polyacrylamide gel electrophoresis, and transferred onto a polyvinylidene fluoride membrane (Millipore, MA, USA). The membranes were then blocked in nonfat milk for $1 \mathrm{~h}$, and incubated with the primary antibodies against NF- $\mathrm{kB}$ p-p65 and NFкB p65 (1:1000; Cell Signaling Technology, Beverly, MA, USA); COX-2 (1:1000; Abcam, Cambridge, MA, USA); ICAM-1 (1:500); Arginase-1 (1:1000); TGF- $\beta$ (1:1000; ); $\beta$-actin (1:5000; Proteintech, Chicago, IL, USA) or histone H3 (1:1000, Abaways, Shanghai, China) at $4{ }^{\circ} \mathrm{C}$ overnight. Following three washes $(10 \mathrm{~min}$ each time) with Tris-buffered saline in tween (TBST), the membranes were incubated with horseradish peroxidase-conjugated anti-rabbit secondary antibody (1:5000; Cell Signaling Technology). Immunoreactive bands were detected using the enhanced chemiluminescence detection system (ECL; Beyotime Institute of Biotechnology, Jiangsu, China), and protein band densities were digitally quantified by Image J software, and normalized to $\beta$-actin or histone $\mathrm{H} 3$.

ELISA for the detection of IL-1 $\beta, I L-6, T N F-\alpha$ and IL-10

Levels of IL-1 $\beta$, IL- 6, TNF- $\alpha$ and IL-10 in the ipsilateral penumbra were measured at $24 \mathrm{~h}$ after reperfusion. Each brain tissue sample was homogenized in phosphate-buffered saline and centrifuged at $10,000 \mathrm{~g}$ at $4^{\circ} \mathrm{C}$ for $10 \mathrm{~min}$. The supernatants from brain tissues were collected and measured using commercially available ELISA kits (Boster, Wuhan, China) according to the manufacturer's instructions. Samples were run in duplicates, and absorbance was read using a microplate reader (BioTek Instruments Inc, VT, USA). 


\section{Cellular Physiology Cell Physiol Biochem 2018;48:42-53 \begin{tabular}{l|l} 
and Biochemistry Published ondine: July 11, 2018 & $\begin{array}{l}\text { (c) } 2018 \text { The Author(s). Published by S. Karger AG, Basel } \\
\text { www.karger.com/cpb }\end{array}$
\end{tabular}}

Wang et al.: A-LA Induces Microglia to M2 Phenotype in Stroke

BV2 cell culture and lipopolysaccharide stimulation

The BV2 microglial cell line was purchased from the Institute of Microbiology, Chinese Academy of Sciences (Beijing, China). BV2 cells exposed to lipopolysaccharide (LPS) for $24 \mathrm{~h}$ were used as a model that mimicked microglial inflammation in humans [26]. Cells were maintained in culture medium containing

Table 1. Primers for RT-qPCR

\begin{tabular}{lcc}
\hline Gene & Forward & Reverse \\
\hline IL-1 $\beta$ & GCAACTGTTCCTGAACTCAACT & ATCTTTTGGGGCGTCAACT \\
TNF- $\alpha$ & CCCTCACACTCAGATCATCTTCT & GCTACGACGTGGGCTACAG \\
IL-6 & TAGTCCTTCCTACCCCAATTTCC & TTGGTCCTTAGCCACTCCTTC \\
Arginase-1 & GAACACGGCAGTGGCTTAAC & TGCTTAGCTCTGTCTGCTTTGC \\
Ym1 & GGAGTAGAGACCATGGCACTGAAC & GACTTGCGTGACTATGAAGCATTG \\
TGF- $\beta$ & TGACGTCACTGGAGTTGTACGG & GGTTCATGTCATGGATGGTGC \\
\hline
\end{tabular}
DMEM (Dulbecco's Modified Eagle's Medium; high glucose, Gibco, CA, USA), 10\% heat-inactivated fetal bovine serum (FBS; Gibco, CA, USA) and 1\% penicillin/ streptomycin at $37{ }^{\circ} \mathrm{C}$ in a humidified incubator under a $95 \% / 5 \%(\mathrm{v} / \mathrm{v})$ mixture of air and $\mathrm{CO}_{2}$. The cultured cells were pretreated for $2 \mathrm{~h}$ with different concentrations of $\alpha$-LA $((0,1,10,100 \mu \mathrm{M} / \mathrm{L}$; Sigma-Aldrich, M0, USA) followed by stimulation with $1 \mu \mathrm{g} / \mathrm{mL}$ LPS (Sigma-Aldrich) for $24 \mathrm{~h}$.

\section{Immunofluorescence staining}

The effect of $\alpha$-LA on the activation of NF- $\kappa B$ was determined using immunofluorescence. BV2 cells were fixed in $4 \%$ paraformaldehyde for $15 \mathrm{~min}$, and rinsed with PBS. The cells were permeabilized in Triton $\mathrm{X}-100(0.5 \%, 200 \mu \mathrm{l})$ for $20 \mathrm{~min}$ at room temperature. Adherent cells were used for immunofluorescence staining. Briefly, cells were incubated with anti-Iba1 or anti-NF- $\kappa$ B p65 antibody (both at 1:100; Abcam) at $4{ }^{\circ} \mathrm{C}$ overnight. The next day, cells were washed with PBS, and incubated with anti-rabbit-IgG or antiGoat-IgG (1:1000; Invitrogen, CA, USA) for $1 \mathrm{~h}$ at room temperature. Subsequently, cells were washed in PBS-T and counterstained with Hoechst (1:10000; Invitrogen) for 5 min in the dark. For mounting, anti-fade solution (Dako, CA, USA) was added to the plates. All images were viewed and recorded using a fluorescence microscope (CKX41, Tokyo, Japan).

\section{Quantitative RT-PCR}

We evaluated the effect of $100 \mu \mathrm{M} \alpha$-LA on M1/M2 microglial phenotype using quantitative real-time PCR (qRT-PCR). Total RNA samples BV2 were isolated using TRIzol Reagent (Life Technologies). cDNA synthesis was carried out using the PrimeScript II 1st strand cDNA Synthesis Kit (Takara, Dalian, China) and qPCR was performed using an SYBR Green kit (Takara) according to manufacturer's instructions. We repeated all reactions at least three times with the following conditions: denaturing at $95^{\circ} \mathrm{C}$ for $30 \mathrm{~s}$, followed by 40 cycles of $95^{\circ} \mathrm{C}$ for $5 \mathrm{~s}$ and $60^{\circ} \mathrm{C}$ for $30 \mathrm{~s}$. The data were analyzed by the comparative threshold cycle (Ct) method, and the results were expressed as fold change normalized to GAPDH. The primer sequences were reported in Table 1.

\section{Statistical analysis}

Data of Garcia scores are presented as median (25th, 75th percentiles) and analyzed using the KruskalWallis test. A Mann-Whitney $U$ analysis was performed when the overall $P$ value was significant. Other data are presented as the mean \pm SD and analyzed using one-way analysis of variance (ANOVA) with Bonferroni correction for post hoc comparison between multiple experimental groups. $P<0.05$ was considered statistically significant. Statistical analyses were performed using SPSS software (SPSS Inc., Chicago, IL, USA).

\section{Results}

$\alpha$-LA reduced infarct volume after $M C A O$

The effect of different doses of $\alpha$-LA on infarct size was evaluated $24 \mathrm{~h}$ after reperfusion. The results exhibited significantly greater infarct volume in the control group (47.6 $\pm 8.9 \%)$ compared to LA20 $(32.7 \pm 8.7 \%)$ and LA40 $(22.2 \pm 7.9 \%)$ groups $(P<0.05$; Fig. 1 A and $1 \mathrm{~B})$. Moreover, LA40 groups had significantly alleviated infarct volumes compared with the LA20 group $(P<0.05)$.

$\alpha$-LA improved neurological outcome after MCAO

After $24 \mathrm{~h}$ of MCAO, rats were evaluated for the extent of neurological deficits and graded from 0-18 using the Garcia score system. As shown in Fig. 2, no neurological deficits 
Fig. 1. $\alpha$-LA reduced infarct volume after MCAO. A. Representative samples of brain stained with TTC after $24 \mathrm{~h}$ reperfusion. B. Infarction volume (white area) is shown for each group. $\mathrm{n}=10$ in each group. LA20, $\alpha$-lipoic acid $20 \mathrm{mg} / \mathrm{kg}$ group; LA40, $\alpha$-lipoic acid $40 \mathrm{mg} / \mathrm{kg}$ group; * $\mathrm{P}<0.05$ vs. Control group, \# $\mathrm{P}<0.05$ vs. LA20 group.

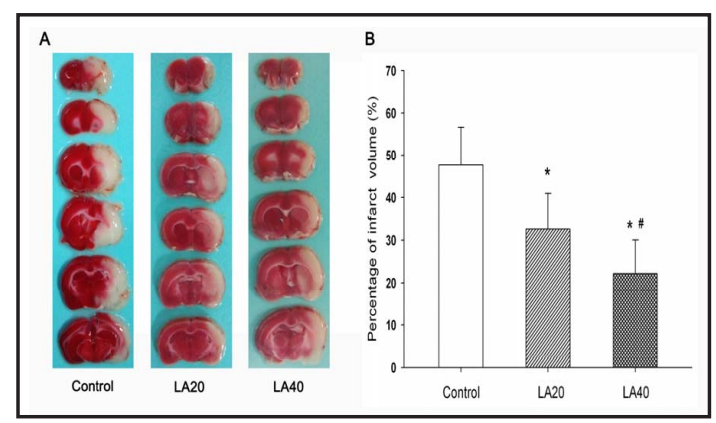

Fig. 2. $\alpha$-LA improved neurological outcome after MCAO. After $24 \mathrm{~h}$ of MCAO, rats were evaluated for the extent of neurological deficits and graded from 0-18 using the Garcia score system. $n=10$ in each group. LA20, $\alpha$-lipoic acid $20 \mathrm{mg} / \mathrm{kg}$ group; LA40, $\alpha$-lipoic acid $40 \mathrm{mg} / \mathrm{kg}$ group; ${ }^{*} \mathrm{P}<0.05$ vs. Sham group, ${ }^{\#} \mathrm{P}<0.05$ vs. Control group, ${ }^{\dagger} \mathrm{P}<0.05$ vs. LA2 0 group.

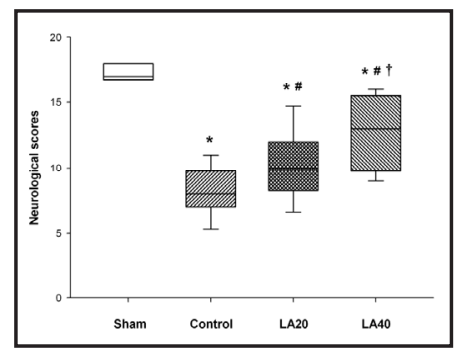

Fig. 3. $\alpha$-LA modulated microglia M1/M2 polarization after MCAO. A. Expression of COX-2 and ICAM-1 in brain tissue samples from each group. B. Expression of Arginase- 1 and TGF- $\beta$ in brain tissue samples from each group. Representative immunoblots are shown on the top of each panel, whereas quantitative results are shown in the respective lower panel. $\mathrm{n}=10$ in each group. LA20, $\alpha$-lipoic acid $20 \mathrm{mg} /$ $\mathrm{kg}$ group; LA40, $\alpha$-lipoic acid $40 \mathrm{mg} / \mathrm{kg}$ group; ${ }^{\mathrm{P}}<0.05$ vs. Sham group, ${ }^{*} \mathrm{P}<0.05$ vs. Control group, ${ }^{\dagger} \mathrm{P}<0.05$ vs. LA20 group.

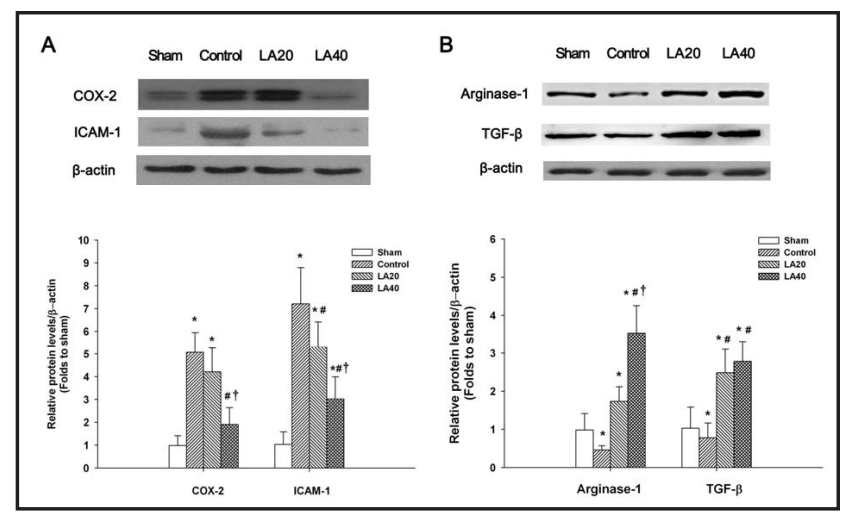

were observed in the sham group $(17[17,18])$, however the Garcia scores in the control $(8[7,10])$, LA20 $(10[8,12])$ and LA40 groups $(13[10,15])$ were significantly lower $(P<$ $0.05)$. Additionally, rats treated with 20 and $40 \mathrm{mg} / \mathrm{kg}$ of $\alpha$-LA showed greatly improved neurological behavior compared with rats in the control group $(P<0.05)$. Moreover, rats in the LA40 group showed better neurological outcome than LA20 group $(P<0.05$; Fig. 2).

\section{$\alpha$-LA modulated microglia M1/M2 polarization after $2 \mathrm{~h}$ of $M C A O$}

The markers of activated microglial phenotypes after stroke were examined using western blot. As shown in Fig. 3A, COX-2 expression increased significantly in the control (5.1 \pm 0.9 fold of sham) and LA20 (4.2 \pm 1.0 fold of sham) groups compared with the sham group $(P<0.05)$. Interestingly, COX-2 expression in the LA40 group $(1.9 \pm 0.7$ fold of sham) was significantly lower than in the control and LA20 groups $(P<0.05)$. Similarly, the expression of ICAM-1 in control (7.2 \pm 1.6 fold of sham), LA20 (5.3 \pm 1.1 fold of sham) and LA40 (3.0 \pm 0.9 ) groups were significantly higher than in the sham group $(P<0.05)$. The expression of ICAM-1 significantly decreased in the LA20 and LA40 groups compared with the control group $(P<0.05)$, and was further lower in the LA40 group $(P<0.05$, Fig. 4B).

Arginase- 1 and TGF- $\beta$ are markers of the activated microglial M2 phenotype. As shown in Fig. 3B, Arginase-1 expression decreased significantly in the control group $(0.46 \pm 0.22$ fold of sham), while increased in the LA20 (1.73 \pm 0.38 fold of sham) and LA40 (3.53 \pm 0.52 
fold of sham) groups compared with the sham group $(P<0.05)$. Compared with the LA20 group, the expression of Arginase- 1 was further decreased in LA40 group $(P<0.05)$. The expression of TNF- $\beta$ in control ( $0.78 \pm 0.39$ fold of sham) was significantly lower than in the sham group. While the levels of TNF- $\beta$ in LA20 (2.48 \pm 0.62 fold of sham) and LA40 (2.78 \pm $0.53)$ groups were significantly higher than in the sham group (both $P<0.05$ ).

$\alpha$-LA modulated the expression of pro-inflammatory and anti-inflammatory cytokines after MCAO

As shown in Fig. 4A, we found significantly increased levels of IL- $1 \beta$ in the control $(40.8$ $\pm 8.2 \mathrm{pg} / \mathrm{mg})$ and LA20 $(30.4 \pm 3.3 \mathrm{pg} / \mathrm{mg})$ groups compared with the sham group $(16.0 \pm 3.9$ $\mathrm{pg} / \mathrm{mg}$ ) (both $P<0.05)$. However, LA20 and LA40 $(24.7 \pm 3.6 \mathrm{pg} / \mathrm{mg})$ groups had significantly reduced IL-1 $\beta$ levels than the control group (both $P<0.05$ ).

The levels of IL- 6 were significantly elevated in control $(53.0 \pm 8.8 \mathrm{pg} / \mathrm{mg})$ and LA20 $(37.3 \pm 4.5 \mathrm{pg} / \mathrm{mg})$ groups compared with the sham group $(17.0 \pm 4.2 \mathrm{pg} / \mathrm{mg})(P<0.05)$. Decreased IL-6 levels were observed in LA20 and LA40 $(25.4 \pm 3.8 \mathrm{pg} / \mathrm{mg})$ groups compared with the control group (both $P<0.05)$, with potently lower levels in the LA40 group $(P<$ 0.05 , Fig. 4B).

Furthermore, the levels of TNF- $\alpha$ in control $(32.6 \pm 5.6 \mathrm{pg} / \mathrm{mg})$ and LA20 $(25.2 \pm 1.1 \mathrm{pg} /$ $\mathrm{mg}$ ) groups increased significantly compared with sham group $(10.3 \pm 2.8 \mathrm{pg} / \mathrm{mg}$ ) (both $P$ $<0.05)$; whereas, the levels were significantly lower in LA20 and LA40 $(15.2 \pm 4.3 \mathrm{pg} / \mathrm{mg})$ groups than the control group (both $P<0.05$ ). The TNF- $\alpha$ was further decreased in LA40 group compared with LA20 group $(P<0.05$, Fig. 4C).

Different from these pro-inflammatory cytokines, the expression of IL-10 was increased in control $(16.25 \pm 3.59 \mathrm{pg} / \mathrm{mg}), \mathrm{LA} 20(37.10 \pm 8.51 \mathrm{pg} / \mathrm{mg})$ and LA40 (42.87 $\pm 8.92 \mathrm{pg} / \mathrm{mg})$ groups compared with the sham group $(4.73 \pm 1.24$, all $P<0.05)$. Compared with control group, the levels of IL-10 were significantly increased in LA20 and LA40 group (both $P<$ 0.05 , Fig. 4D).

\section{$\alpha$-LA attenuated NF- $\kappa B$ activation after MCAO}

Using western blotting, we evaluated the effect of $\alpha$-LA on the expression of activated NF- $\kappa B$ that was induced by MCAO. The results showed significantly elevated levels of phosphorylated NF- $\kappa B$ p65 (NF- $\kappa B$ p-p65) in brain tissue samples of control ( $4.3 \pm 0.8$ fold of sham) and LA20 (2.8 \pm 0.9 fold of sham) groups $(P<0.05$; Fig. 3A). However, treatment with 20 or $40 \mathrm{mg} / \mathrm{kg} \alpha$-LA ( $1.5 \pm 0.6$ fold of sham) significantly inhibited the expression of NF- $\kappa B$ p-p65 compared with the control group (both $P<0.05$ ). Also, NF- $\kappa B$ p-p65 expression decreased further in LA40 group compared with LA20 group $(P<0.05$; Fig. 5A).

Furthermore, we detected the expression of NF- $\mathrm{KB}$ p 65 in the nuclear and cytosolic fractions of the brain tissue samples. We quantified the nucleus/cytoplasm ratio of NF- $\kappa \mathrm{B}$ $\mathrm{p} 65$, and found the increased ratio in the control ( $4.4 \pm 0.6$ fold of sham) and LA20 groups

Fig. 4. $\alpha$-LA modulated the expression of proinflammatory and anti-inflammatory cytokines after MCAO. The effects of $\alpha$-LA on the expression of IL-1 $\beta$, IL-6, TNF- $\alpha$ and IL-10 in the ipsilateral penumbra post MCAO were evaluated using ELISA. $\mathrm{n}=10$ in each group. LA20, $\alpha$-lipoic acid $20 \mathrm{mg} /$ kg group; LA40, $\alpha$-lipoic acid $40 \mathrm{mg} / \mathrm{kg}$ group; * $\mathrm{P}<0.05$ vs. Sham group, ${ }^{\#} \mathrm{P}<0.05$ vs. Control group, $+\mathrm{P}<0.05$ vs. LA2 0 group.

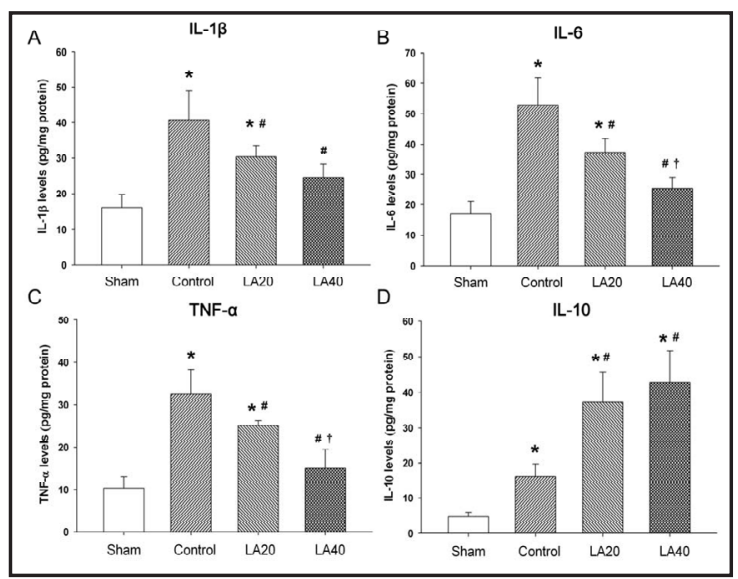


Fig. 5. $\alpha$-LA attenuated NF$\kappa \mathrm{B}$ activation after MCAO. A. Expression of phosphorylated NF$\kappa \mathrm{B}$ p65 in brain tissue samples from each group. B. Nucleus/ Cytoplasm ratio of $N F-\kappa B$ p65 in brain tissue samples from each group. Representative immunoblots are shown on the top of each panel, whereas quantitative results are shown in the respective lower panel. $\mathrm{n}=10$ in each group. LA20, $\alpha$-lipoic acid $20 \mathrm{mg} / \mathrm{kg}$ group; LA40, $\alpha$-lipoic acid $40 \mathrm{mg} /$ kg group; ${ }^{*} \mathrm{P}<0.05$ vs. Sham group, ${ }^{\#} \mathrm{P}<0.05$ vs. Control group,${ }^{\dagger} \mathrm{P}<0.05$ vs. LA20 group.

Fig. 6. $\alpha$-LA inhibited the expression of M1 markers and increased activation of the M2 markers in LPS-stimulated BV2 microglia. The effects of $\alpha$-LA on the mRNA expressions of IL-1 $\beta$, TNF- $\alpha$, IL-6, Arginase- $1, \mathrm{Ym} 1$, and TGF- $\beta$ in the LPS-stimulated BV2 microglia were evaluated using quantitative RT-PCR. Data are representative of three independent experiments and are shown as relative mRNA. * $\mathrm{P}<0.05$ vs. Control group, ${ }^{\#} \mathrm{P}<0.05$

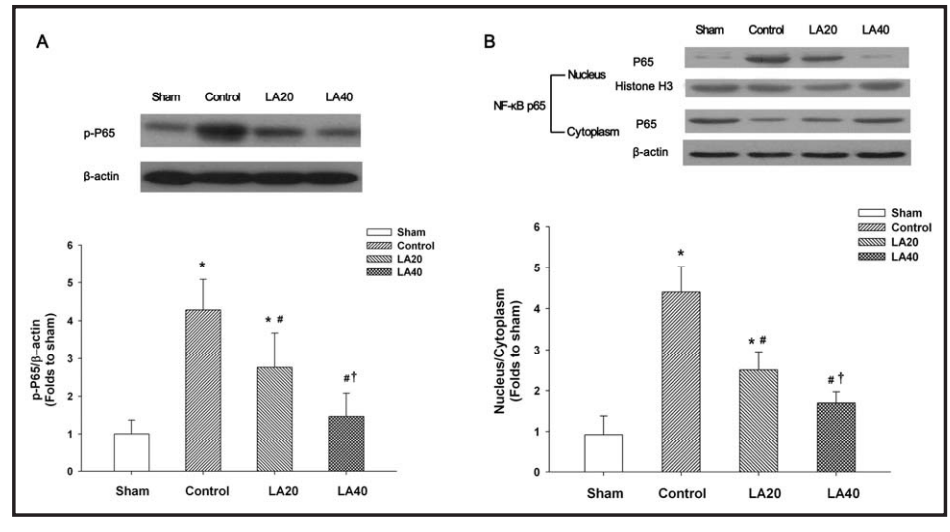
vs. LPS group.

( $2.5 \pm 0.4$ fold of sham) compared with the sham group (both $P<0.05)$. The ratio in LA20 and LA40 (1.7 \pm 0.3 fold of sham) groups was significantly lower than the control group (both $P$ $<0.05)$. Additionally, reduced NF- $\kappa \mathrm{B}$ p65 nucleus/cytoplasm ratio was observed in the LA40 group compared with the LA20 group $(P<0.05$; Fig. 5B).

$\alpha$-LA inhibited the expression of M1 markers and increased activation of the M2 markers in LPS-stimulated BV2 microglia

The mRNA expressions of M1 and M2 activation markers are shown in Fig. 6. The levels of IL-1 $\beta$ (4.02 \pm 0.83 fold of control), TNF- $\alpha$ (4.02 \pm 0.73 fold of control) and IL-6 (6.08 \pm 0.80 fold of control) were consistently increased after the LPS stimulation (all $P<0.05$ ). These increases were significantly inhibited in the $\alpha$-LA group (1.60 \pm 0.39 fold, $1.68 \pm 0.54$ fold, $1.88 \pm 0.66$ fold, respectively, all $P<0.05$ ). In contrast, the M2 polarization markers Arginase- 1 ( $0.38 \pm 0.27$ fold of control) and IGF- $\beta(0.55 \pm 0.15$ fold of control) were found to be decreased after the LPS stimulation (both $P<0.05$ ). Compared with control and LPS groups, Arginase- 1 (2.05 \pm 0.49 fold), Ym1 (2.67 \pm 0.50 fold $)$ and IGF- $\beta(2.03 \pm 0.30$ fold $)$ were found to be strongly upregulated after $\alpha$-LA treatment (all $P<0.05$ ).

$\alpha$-LA suppressed the nuclear translocation of NF- $\kappa B$ in LPS-stimulated BV2 microglia

The effect of $\alpha$-LA on NF- $\kappa B$ activation in BV2 cells stimulated with LPS $(1 \mu \mathrm{g} / \mathrm{ml})$ was determined using immunofluorescence. As shown in Fig. 7, enhanced nuclear translocation of NF- $\kappa$ B p65 was detected in LPS stimulated BV2 cells. Pretreatment with $\alpha$-LA suppressed NF-кB p65 nuclear translocation. Compared with the control group $(6.18 \pm 3.08 \%)$, the percentage of nuclear translocation of NF- $\mathrm{KB}$ p 65 was significantly increased in LPS group 
Fig. 7. $\alpha$-LA suppressed the nuclear translocation of $\mathrm{NF}-\kappa \mathrm{B}$ in LPS-stimulated BV2 microglia. The effect of $\alpha$-LA on the nuclear translocation of NF- $\kappa$ B in BV2 cells stimulated with LPS $(1 \mu \mathrm{g} / \mathrm{ml})$ was determined using immunofluorescence. A. Cells were stained with Hoechst (blue), NF- $\kappa$ B p65 (red) or Iba1 (green) antibody. B. The percentage of NF- $\kappa B$ p65/Hoechst is quantified by the intensity of Iba $1^{+}$

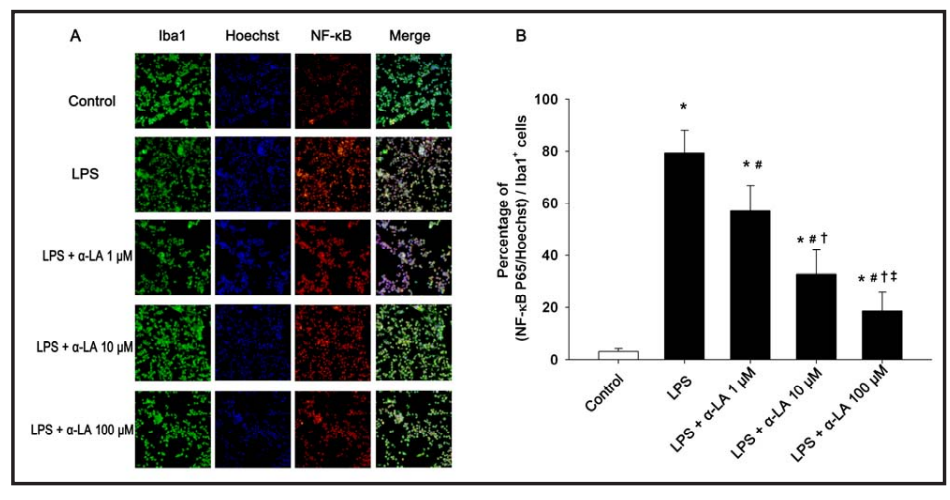
immunofluorescence. All images were viewed and recorded using a fluorescence microscope. The images are representative of three independent experiments performed in triplicates. ${ }^{*} \mathrm{P}<0.05$ vs. Control group, ${ }^{\#} \mathrm{P}<0.05$ vs. LPS group, ${ }^{\dagger} \mathrm{P}<0.05$ vs. LPS $+\alpha$-LA $1 \mu \mathrm{M}$ group; ${ }^{\ddagger} \mathrm{P}<0.05$ vs. LPS $+\alpha$-LA $10 \mu \mathrm{M}$ group. Magnification: $400 \times$.

$(79.37 \pm 8.55 \%, P<0.05)$. This increase was significantly inhibited in $\alpha$-LA $1 \mu \mathrm{M}(57.04 \pm$ 9.83\%), $10 \mu \mathrm{M}$ and $100 \mu \mathrm{M}$ groups respectively (all $P<0.05$ ). Compared with $\alpha$-LA $1 \mu \mathrm{M}$ group, the percentage of nuclear translocation of NF- $\kappa B$ p65 was significantly decreased in $\alpha$-LA $10 \mu$ M group $(32.91 \pm 9.20 \%, P<0.05)$ and further decreased in $\alpha$-LA $100 \mu$ M group $(18.63 \pm 7.37 \%, P<0.05)$.

\section{Discussion}

In the present study focusing on anti-inflammation, we found that administration of $\alpha$-LA in a rat model of MCAO resulted in decreased infarction volume and significantly improved neurological outcome in a dose-dependent manner, indicating that $\alpha$-LA exhibited neuroprotective effects in vivo. In addition, it modulated the polarization of microglia M1/M2, decreased the expression of pro-inflammatory cytokines, increased the antiinflammatory cytokines, and attenuated NF- $\mathrm{KB}$ activation after MCAO. Phenotypic analysis of LPS-stimulated BV2 microglia showed that $\alpha$-LA significantly inhibited the expression of M1 markers and increased activation of the M2 markers. Moreover, $\alpha$-LA suppressed the nuclear translocation of NF-кB in LPS-stimulated BV2 microglia.

Microglia is the major immune cell involved in cerebral ischemia and reperfusion injury [16]. In stroke, microglia becomes activated in the penumbra when reperfusion begins and starts to expand cellular protrusions towards the adjacent blood vessels. The morphological and phenotypic changes in the native microglia in the brain represent different roles in the injured brain. The M1 phenotype is responsible for increased pro-inflammatory cytokines, while M2 phenotype plays an important regulatory role to inhibit inflammation and promote tissue remodeling [27]. Therefore, modulation of microglial M1/M2 phenotype may represent an innovative method for the treatment of stroke. In our in vivo study, we found that after $\alpha$-LA treatment, the increased microglia M1 markers COX2 and ICAM- 1 were reduced, whereas the M2 markers Arginase- 1 and TGF- $\beta$ were upregulated significantly. At the same time, the levels of proinflammatory factors were inhibited remarkably in a dosedependent manner after $\alpha$-LA treatment, whereas IL-10 that associated with alternative activation of microglia was upregulated.

To further investigate the effects of $\alpha$-LA on modulating microglia M1/M2 polarization, we utilized BV2 cell line stimulated with LPS as an in vitro model of microglial inflammation [26]. Selected expression of M1/M2 activation markers was quantified by qPCR. In line with the in vivo study, LPS induced IL-1 $\beta$, TNF- $\alpha$, IL- 6 expression and inhibited Arginase-1, TGF- $\beta$ expression. $\alpha$-LA treatment resulted in the downregulation of the M1 markers IL-1 $\beta$, TNF- $\alpha$ 
and IL-6. In contrast, the M2 polarization markers Arginase-1, Ym1 and TGF- $\beta$ were found to be strongly upregulated compared with the LPS stimulation. All of these results in vivo and in vitro studies indicated that $\alpha$-LA exerts neuroprotective effects by inducing the polarization of microglia to the M2 phenotype.

Previous studies indicated that microglia show a transient M2 phenotype in the ischemic penumbra and express high levels of anti-inflammatory factors to improve neuron survival and reduce cerebral infarct volume within $24 \mathrm{~h}$ after ischemic stroke $[28,29]$. Then, activated microglia shift to the M1 phenotype and worsen tissue destruction and neurological dysfunction. Therefore, the regulation of microglia M1/M2 phenotype at the early stage is crucial to protect the brain against damage induced by stroke. The results of phenotypic analysis of LPS-stimulated BV2 microglia revealed that $\alpha$-LA significantly inhibited M1 transformation and promoted the M2 transformation of microglia at the early stage. These findings suggest that $\alpha$-LA is a possible therapeutic candidate for ischemic stroke through regulating the polarization of microglia.

$\mathrm{NF}-\kappa \mathrm{B}$ is a key transcription factor that has been shown to regulate the expression of pro-inflammatory factors involved in activated microglia [30]. A growing body of evidence suggests that inhibition of NF- $\mathrm{KB}$ can directly or indirectly promote neuroprotective M2 polarization of the microglia [31, 32]. Dang et al [31]. found that papaverine promoted the transformation of activated BV2 cells from M1/M2b to a neuroprotective M2a phenotype through NF- $\kappa B$ signaling pathway. Su et al [32]. also demonstrated that mycoepoxydiene inhibited the activation of BV2 microglia stimulated by LPS through suppression of NF- $\kappa$ B. Therefore, we investigated whether the effect of $\alpha$-LA on modulating the polarization of microglia was associated with the NF- $\mathrm{kB}$. Our in vivo study showed that MCAO significantly enhanced the activation of NF- $\kappa \mathrm{B}$, while $\alpha$-LA remarkably inhibited the phosphorylation and nuclear translocation of NF- $\kappa B$ in a dose-dependent manner. Furthermore, we investigated the effect of $\alpha$-LA on LPS-induced NF- $\kappa B$ nuclear translocation using immunofluorescence assay. We found that $\alpha$-LA dose-dependently inhibited LPS-induced NF- $\kappa B$ nuclear translocation. This was accompanied by alleviation of classically activated M1 markers and promotion of alternatively activated M2 markers.

The molecular basis of microglia polarization remains unclear. The NF- $\mathrm{KB}$ family of transcription factors is thought to be involved in macrophage M1 and M2 signaling [33] and have been implicated in microglial activation [34]. The NF- $\kappa B$ family includes five members (p50, p52, p65, c-Rel, and Rel-B). In the nervous system, the phosphorylation of p65 helps stabilized NF- $\kappa B$ complex in the nucleus for gene expression and thereby is widely used as an indicator of NF- $\kappa \mathrm{B}$ activation [30]. In addition, previous studies have reported that p65 phosphorylation induced microglia polarization $[35,36]$. So, we examined the phosphorylation of p65 in vivo and in the BV2 cell to investigate whether the phosphorylation of p65 participates in $\alpha$-LA regulating NF- $\kappa B$ activation. The result indicated that after MCAO, the phosphorylation of p65 was increased and translocated to nuclei while $\alpha$-LA reversed this effect. Moreover, the nuclear translocation of p65 in LPS-stimulated BV2 cells was significantly reduced by $\alpha$-LA treatment, indicating that phosphorylation of p65 participated in $\alpha$-LA regulating NF- $\kappa B$ activation. Taken together, these results suggest that inhibition of NF- $\kappa B$ activation by $\alpha$-LA via regulating phosphorylation and nuclear translocation of p65 may be the mechanism responsible for modulating microglia M1/M2 polarization.

Oxidative stress also plays an important role in ischemic stroke pathogenesis. A landmark study by Salzano et al. has demonstrated a causal link between oxidative stress and inflammatory activation [37]. The release of redox proteomics identified peroxiredoxin 2 from macrophages can enable induction of inflammatory responses and trigger the production of TNF- $\alpha$. The anti-inflammatory effects of $\alpha$-LA shown in this study, therefore, might be a downstream effect of reduced oxidative stress. It thus remains for future studies to further investigate whether the anti-inflammatory effects of $\alpha$-LA is independent of oxidative stress or not.

This study has several limitations. Firstly, since the single dose of $\alpha$-LA was used in our study, further studies about the effects of the delayed and prolonged application 


\section{Cellular Physiology Cell Physiol Biochem 2018;48:42-53 \begin{tabular}{l|l} 
and Biochemistry Published 10.1159/000491661 2018 & $\begin{array}{l}\text { (c) } 2018 \text { The Author(s). Published by S. Karger AG, Basel } \\
\text { www.karger.com/cpb }\end{array}$ \\
\hline
\end{tabular}}

Wang et al.: A-LA Induces Microglia to M2 Phenotype in Stroke

of $\alpha$-LA are needed. The long-term neurological effect conducted by $\alpha$-LA should also be further examined. Furthermore, LPS was the most common inducer used to study microglia phenotypic polarization, but the LPS model of inflammation is not the most relatable model in regards to ischemic stroke. An oxygen and glucose deprivation, and reperfusion (OGD/R) in vitro model would be better to support the main finding of this study. Lastly, though our findings demonstrated improvement of early neurological function after treatment with $\alpha$-LA in stroke, the results should be taken in caution due to the limitation of the study and further studies are needed to confirm these findings.

In conclusion, the current study revealed that $\alpha$-LA alleviated ischemic stroke-induced brain infarction and neurological deficits. The neuroprotective effect of $\alpha$-LA may involve inhibition of cerebral ischemia reperfusion injury-induced inflammation reactions by modulating microglia M1/M2 polarization. The potential mechanism of $\alpha$-LA might be mediated by inhibition of NF- $\kappa \mathrm{B}$ activation via regulating phosphorylation and nuclear translocation of p65.

\section{Acknowledgements}

This study was supported by grants from the National Natural Science Foundation of China (81372026). The funders had no role in study design, data collection and analysis, decision to publish, or preparation of the manuscript.

Bing Zhang designed the study and revised the first draft. Qingqing Wang, Chengmei Lv and Yongxin Sun carried out most of the experiment. Qingqing Wang managed the retrieval of most references and contributed to the writing of the first draft. Xu Han, Shan Wang, Zhengchun Mao and Yanming Xin provided assistance in the conduction of the study.

\section{Disclosure Statement}

The authors declare no Disclosure Statement.

\section{References}

1 Iadecola C, Anrather J: The immunology of stroke: from mechanisms to translation. Nat Med 2011;17:796808.

2 Lyerly MJ, Albright KC, Boehme AK, Bavarsad Shahripour R, Houston JT, Rawal PV, Kapoor N, Alvi M, Sisson A: Safety of protocol violations in acute stroke tPA administration. J Stroke Cerebrovasc Dis 2014;23:855860.

-3 Knecht T, Story J, Liu J, Davis W, Borlongan CV, Dela Peña IC: Adjunctive Therapy Approaches for Ischemic Stroke: Innovations to Expand Time Window of Treatment. Int J Mol Sci 2017;18:pii: E2756.

4 Venkat P, Shen Y, Chopp M, Chen J: Cell-based and pharmacological neurorestorative therapies for ischemic stroke. Neuropharmacology 2018;134:310-322.

-5 Konrad T, Vicini P, Kusterer K, Höflich A, Assadkhani A, Böhles HJ, Sewell A, Tritschler HJ, Cobelli C, Usadel $\mathrm{KH}$ : alpha-Lipoic acid treatment decreases serum lactate and pyruvate concentrations and improves glucose effectiveness in lean and obese patients with type 2 diabetes. Diabetes Care 1999; 22:280-287.

6 Choi KH, Park MS, Kim JT, Kim HS, Kim JH, Nam TS, Choi SM, Lee SH, Kim BC, Kim MK, Cho KH: Lipoic Acid Use and Functional Outcomes after Thrombolysis in Patients with Acute Ischemic Stroke and Diabetes. PLoS One 2016;11:e0163484.

7 Lv C, Maharjan S, Wang Q, Sun Y, Han X, Wang S, Mao Z, Xin Y, Zhang B. $\alpha$-Lipoic Acid Promotes Neurological Recovery After Ischemic Stroke by Activating the Nrf2/HO-1 Pathway to Attenuate Oxidative Damage. Cell Physiol Biochem 2017;43:1273-1287.

$>8$ Moura FA, de Andrade KQ dos Santos JC, Goulart MO: Lipoic Acid: its antioxidant and anti-inflammatory role and clinical applications. Curr Top Med Chem 2015;15:458-483. 


\section{Cellular Physiology Cell Physiol Biochem 2018;48:42-53

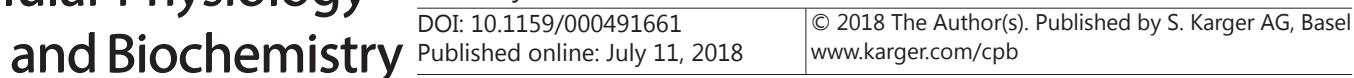

Wang et al.: A-LA Induces Microglia to M2 Phenotype in Stroke

9 Chaudhary P, Marracci G, Galipeau D, Pocius E, Morris B, Bourdette D: Lipoic acid reduces inflammation in a mouse focal cortical experimental autoimmune encephalomyelitis model. J Neuroimmunol 2015;289:6874.

10 Choi KH, Park MS, Kim HS, Kim KT, Kim HS, Kim JT, Kim BC, Kim MK, Park JT, Cho KH: Alpha-lipoic acid treatment is neurorestorative and promotes functional recovery after stroke in rats. Mol Brain 2015;8:9.

11 Chang P, Liu J, Yu Y, Cui SY, Guo ZH, Chen GM, Huang Q, Liu ZG. Alpha-Lipoic Acid Suppresses Extracellular Histone-Induced Release of the Infammatory Mediator Tumor Necrosis Factor- $\alpha$ by Macrophages. Cell Physiol Biochem 2017;42:2559-2568.

12 Mizuma A, Yenari MA: Anti-Inflammatory Targets for the Treatment of Reperfusion Injury in Stroke. Front Neurol 2017;8:467.

13 Jin R, Yang G, Li G: Inflammatory mechanisms in ischemic stroke: role of inflammatory cells. J Leukoc Biol 2010;87:779-789.

-14 Raza SS, Khan MM, Ahmad A, Ashafaq M, Islam F, Wagner AP, Safhi MM, Islam F: Neuroprotective effect of naringenin is mediated through suppression of NF- $\kappa B$ signaling pathway in experimental stroke. Neuroscience 2013;230:157-171.

15 Dheen ST, Kaur C, Ling EA: Microglial activation and its implications in the brain diseases. Curr Med Chem 2007;14:1189-1197.

16 Xiong XY, Liu L, Yang QW: Functions and mechanisms of microglia/macrophages in neuroinflammation and neurogenesis after stroke. Prog Neurobiol 2016;142:23-44.

17 Zhao SC, Ma LS, Chu ZH, Xu H, Wu WQ, Liu F: Regulation of microglial activation in stroke. Acta Pharmacol Sin 2017;38:445-458.

18 Kilkenny C, Browne W, Cuthill IC, Emerson M, Altman DG; NC3Rs Reporting Guidelines Working Group: Animal research: reporting in vivo experiments: the ARRIVE guidelines. Br J Pharmacol 2010;160:15771579.

19 McGrath JC, Drummond GB, McLachlan EM, Kilkenny C, Wainwright CL: Guidelines for reporting experiments involving animals: the ARRIVE guidelines. Br J Pharmacol 2010;160:1573-1576.

20 Belayev L, Alonso OF, Busto R, Zhao W, Ginsberg MD: Middle cerebral artery occlusion in the rat by intraluminal suture. Neurological and pathological evaluation of an improved model. Stroke 1996;27:16161622.

-21 Sutherland BA, Buchan AM: Alteplase treatment does not increase brain injury after mechanical middle cerebral artery occlusion in the rat. J Cereb Blood Flow Metab 2013;33:e1-e7.

22 Ikeda-Matsuo Y, Ota A, Fukada T, Uematsu S, Akira S, Sasaki Y: Microsomal prostaglandin E synthase-1 is a critical factor of stroke-reperfusion injury. Proc Natl Acad Sci U S A 2006;103:11790-11795.

23 Garcia JH, Wagner S, Liu KF, Hu XJ: Neurological deficit and extent of neuronal necrosis attributable to middle cerebral artery occlusion in rats. Statistical validation. Stroke 1995;26:627-634.

24 Ashwal S, Tone B, Tian HR, Cole DJ, Pearce WJ: Core and penumbral nitric oxide synthase activity during cerebral ischemia and reperfusion. Stroke 1998;29:1037-1046.

25 Yu Z, Zhou D, Bruce-Keller AJ, Kindy MS, Mattson MP: Lack of the p50 subunit of nuclear factor-kappaB increases the vulnerability of hippocampal neurons to excitotoxic injury. J Neurosci 1999;19:8856-8865.

-26 Hwang SY, Shin JH, Hwang JS, Kim SY, Shin JA, Oh ES, Oh S, Kim JB, Lee JK, Han IO: Glucosamine exerts a neuroprotective effect via suppression of inflammation in rat brain ischemia/reperfusion injury. Glia 2010;58:1881-1892.

27 Hu X, Li P, Guo Y, Wang H, Leak RK, Chen S, Gao Y, Chen J: Microglia/macrophage polarization dynamics reveal novel mechanism of injury expansion after focal cerebral ischemia. Stroke 2012;43:3063-3070.

28 Bell-Temin H, Culver-Cochran AE, Chaput D, Carlson CM, Kuehl M, Burkhardt BR, Bickford PC, Liu B, Stevens SM Jr: Novel Molecular Insights into Classical and Alternative Activation States of Microglia as Revealed by Stable Isotope Labeling by Amino Acids in Cell Culture (SILAC)-based Proteomics. Mol Cell Proteomics 2015;14:3173-3184.

29 Xiang B, Xiao C, Shen T, Li X: Anti-inflammatory effects of anisalcohol on lipopolysaccharide-stimulated BV2 microglia via selective modulation of microglia polarization and down-regulation of NF- $\mathrm{KB}$ p65 and JNK activation. Mol Immunol 2018;95:39-46.

-30 Popiolek-Barczyk K, Mika J: Targeting the Microglial Signaling Pathways: New Insights in the Modulation of Neuropathic Pain. Curr Med Chem 2016;23:2908-2928. 


\section{Cellular Physiology Cell Physiol Biochem 2018;48:42-53 \begin{tabular}{l|l} 
DOI: 10.1159/000491661 & $\begin{array}{l}\text { O 2018 The Author(s). Published by S. Karger AG, Basel } \\
\text { www.karger.com/cpb }\end{array}$
\end{tabular} \\ Wang et al.: A-LA Induces Microglia to M2 Phenotype in Stroke}

-31 Dang Y, Mu Y, Wang K, Xu K, Yang J, Zhu Y, Luo B: Papaverine inhibits lipopolysaccharide-induced microglial activation by suppressing NF-kB signaling pathway. Drug Des Devel Ther 2016;10:851-859.

-32 Su X, Chen Q, Chen W, Chen T, Li W, Li Y, Dou X, Zhang Y, Shen Y, Wu H, Yu C: Mycoepoxydiene inhibits activation of BV2 microglia stimulated by lipopolysaccharide through suppressing NF- $\kappa \mathrm{B}, \mathrm{ERK} 1 / 2$ and tolllike receptor pathways. Int Immunopharmacol 2014;19:88-93.

-33 Porta C, Rimoldi M, Raes G, Brys L, Ghezzi P, Di Liberto D, Dieli F, Ghisletti S, Natoli G, De Baetselier P, Mantovani A, Sica A: Tolerance and M2 (alternative) macrophage polarization are related processes orchestrated by p50 nuclear factor kappaB. Proc Natl Acad Sci U S A 2009;106:14978-14983.

34 Taetzsch T, Levesque S, McGraw C, Brookins S, Luqa R, Bonini MG, Mason RP, Oh U, Block ML: Redox regulation of NF- $\kappa$ B p50 and M1 polarization in microglia. Glia 2015;63:423-440.

35 Nicholas C, Batra S, Vargo MA, Voss OH, Gavrilin MA, Wewers MD, Guttridge DC, Grotewold E, Doseff AI: Apigenin blocks lipopolysaccharide-induced lethality in vivo and proinflammatory cytokines expression by inactivating NF-kappaB through the suppression of p65 phosphorylation. J Immunol 2007;179:7121-7127.

36 Chen J, Yin W, Tu Y, Wang S, Yang X, Chen Q, Zhang X, Han Y, Pi R: L-F001, a novel multifunctional ROCK inhibitor, suppresses neuroinflammation in vitro and in vivo: Involvement of NF-кB inhibition and Nrf2 pathway activation. Eur J Pharmacol 2017;806:1-9.

37 Salzano S, Checconi P, Hanschmann EM, Lillig CH, Bowler LD, Chan P, Vaudry D, Mengozzi M, Coppo L, Sacre S, Atkuri KR, Sahaf B, Herzenberg LA, Herzenberg LA, Mullen L, Ghezzi P: Linkage of inflammation and oxidative stress via release of glutathionylated peroxiredoxin-2, which acts as a danger signal. Proc Natl Acad Sci U S A 2014;111:12157-12162. 